\title{
Hand Digit 4
}

National Cancer Institute

\section{Source}

National Cancer Institute. Hand Digit 4. NCI Thesaurus. Code C52837.

The fourth finger from the radial side of the hand. 